\title{
ATENÇÃO À SAÚDE DO IDOSO POR CUIDADORES INFORMAIS NO CONTEXTO DOMICILIAR: REVISÃO INTEGRATIVA
}

\author{
ELDERLY CARE PROVIDED BY INFORMAL CAREGIVERS IN THE HOME CONTEXT: INTEGRATIVE
}

REVIEW

ATENCIÓN A LA SALUD DEL ANCIANO POR CUIDADORES INFORMALES EN EL CONTEXTO DOMICILIARIO: REVISIÓN INTEGRADORA

\section{Darleia Ferreira Barros dos Santos}

Elane Barreira de Carvalho

Maria do Perpétuo Socorro Santiago do Nascimento

Daniele Martins de Sousa

Herica Emilia Félix de Carvalho

\section{RESUMO}

Este estudo teve por objetivo levantar a produção científica sobre a atenção à saúde do idoso por cuidadores informais Eno contexto domiciliar. Trata-se de revisão integrativa realizada nas bases de dados Lilacs e BDEnf, abrangendo artigos disponiveis em formato eletrônico e texto integral, publicados em português no período de 2011 a 2016. A busca foi realizada em setembro de 2016; da análise dos 14 artigos emergiram 3 categorias temáticas: a) Caracterização do cuidador informal no contexto domiciliar; b) Principais práticas do cuidador informal no contexto domiciliar; e c) Necessidades em educação em saúde dos cuidadores informais de idosos. Constatou-se a necessidade de maior compreensão por parte dos profissionais da saúde acerca das dificuldades relatadas pelos cuidadores, com vistas a conhecer seu perfil e sua rotina e proporcionar melhor qualidade de vida tanto para o cuidador como para o ser humano cuidado.

Palavras-chave: Cuidadores; Assistência a Idosos; Assistência Domiciliar.

\footnotetext{
1. Aluna de graduação em Enfermagem no Centro Universitário UniNovaFapi. Teresina (PI), Brasil.

2. Aluna de graduação em Enfermagem no Centro Universitário UniNovaFapi. Teresina (PI), Brasil.

3. Aluna de graduação em Enfermagem no Centro Universitário UniNovaFapi. Teresina (PI), Brasil.

4. Enfermeira. Mestre em Enfermagem na Universidade Federal do Piauí (UFPI). Teresina (PI), Brasil.

5. Enfermeira. Aluna de mestrado em Enfermagem na UFPI. Teresina (PI), Brasil.
} 


\section{ABSTRACT}

This study aimed to survey the scientific literature on elderly care provided by informal caregivers in the home context. This is an integrative review carried out in the databases LILACS and BDEnf, addressing articles available in electronic format and full text, published in Portuguese within the period from 2011 to 2016. The search was conducted in September 2016; by analyzing 14 articles, 3 thematic categories emerged: a) Informal caregiver's characterization in the home context; b) Main informal caregiver's practices in the home context; and c) Health education needs of the elderly's informal caregivers. We found out the need for greater understanding on the part of health professionals about the difficulties reported by caregivers, in order to know their profile and their routine and afford a better quality of life for both the caregiver and the human being provided with care.

Keywords: Caregivers; Elderly Care; Home Nursing.

\section{RESUMEN}

Este estudio tuvo como objetivo estudiar la literatura científica acerca de la atención al anciano por cuidadores informales en el contexto domiciliario. Esta es una revisión integradora llevada a cabo en las bases de datos LILACS y BDEnf, que aborda los artículos disponibles en formato electrónico y en texto completo, publicados en portugués en el período de 2011 a 2016. La búsqueda se realizó en septiembre de 2016; al analizar 14 artículos, surgieron 3 categorías temáticas: a) Caracterización del cuidador informal en el contexto domiciliario; b) Principales prácticas del cuidador informal en el contexto domiciliario; y c) Necesidades de educación en salud de los cuidadores informales de ancianos. Se constató la necesidad de una mayor comprensión por parte de los profesionales de la salud sobre las dificultades reportadas por los cuidadores, para conocer su perfil y su rutina y proporcionar una mejor calidad de vida tanto para el cuidador como para el ser humano cuidado.

Palabras clave: Cuidadores; Asistencia a los Ancianos; Atención Domiciliaria de Salud.

\section{INTRODUÇÃO}

0 envelhecimento populacional é uma realidade que atinge os mais diversos países do mundo, acarretando significativas modificações em termos de perfil das populações e morbimortalidade ${ }^{1}{ }^{2}$.

Para a Organização Mundial da Saúde (OMS), idoso é toda pessoa com idade $\geq 60$ anos. No Brasil, em uma década, essa população passou de 15,5 milhões de pessoas para 23,5 milhões, correspondendo a $12,3 \%$ da população nacional. A faixa etária com $\geq 80$ anos apresentou maior crescimento, com um contingente de mais de 3 milhões de indivíduos ${ }^{3,4}$.

0 s cuidadores são considerados fundamentais na assistência domiciliar aos idosos, representam o elo entre 0 ser humano cuidado, a família e os serviços de saúde. Existem dois tipos de cuidadores: o formal e o informal. 0 cuidador informal é aquele que desempenha cuidado não profissional, sem receber nenhuma remuneração, ao contrário do cuidador formal. 0 cuidador informal pode ser um familiar, amigo ou vizinho; mas a família, com frequência, desempenha esse papel em equipe 5 .

0 ato de cuidar é enfrentado por muitas famílias sem qualquer experiência anterior. Nessa perspectiva, exige maior atenção, cautela e dedicação. No contexto da responsabilidade à qual os familiares se veem submetidos ao assumir o papel de cuidadores informais, eles podem enfrentar situações de crise, cujos principais sintomas são: constrangimento, estresse, tensão, frustração, fadiga, redução do convívio, depressão e diminuição da autoestima, entre outros. Essa sobrecarga ou tensão pode acarretar problemas psicológicos, físicos, emocionais, sociais e/ou financeiros, afetando o bem-estar do cuidador e do ser humano cuidado ${ }^{6}$.

Assim, para prestar um atendimento domiciliar adequado ao idoso, são necessários cuidadores bem orientados e preparados; para tanto, caberá aos profissionais da saúde oferecer suporte aos cuidadores de idosos por meio da educação em saúde para garantir um cuidado eficaz ${ }^{7}$.

A atenção primária à saúde (APS), no tocante à Estratégia Saúde da Família (ESF), é de suma importância para que a assistência ao idoso atinja o melhor nível possivel. A parceria entre os profissionais da saúde e os cuidadores possibilita a sistematização das tarefas a realizar no próprio domicílio e contribui para uma melhor qualidade de vida do idoso assistido ${ }^{8}$.

Nosso objeto de estudo foi a atenção à saúde do idoso por cuidadores informais no contexto domiciliar; a questão norteadora adotada foi:

- Qual é o status da produção científica sobre atenção à saúde do idoso por cuidadores informais no contexto domiciliar?

Assim, nosso objetivo foi levantar na literatura os aspectos relacionados à atenção à saúde do idoso por 
cuidadores informais no contexto domiciliar.

Esta pesquisa se justifica pela relevância científica de analisar a produção de conhecimento acerca de uma população cujo perfil demográfico e epidemiológico se encontra em mudança no país. A APS deve organizar-se para oferecer apoio aos cuidadores familiares e possibilitar um cuidador contínuo, sistematizado e de qualidade ${ }^{9}$.

\section{MÉTODO}

Trata-se de uma revisão integrativa da literatura - um dos recursos da prática baseada em evidências. Uma revisão integrativa da literatura se fundamenta em 6 etapas: a) elaboração da pergunta norteadora; b) busca ou amostragem na literatura; c) coleta de dados; d) análise crítica dos estudos incluídos; e) discussão dos resultados; e f) apresentação da revisão integrativa ${ }^{10}$.

Aplicou-se a estratégia PICO ${ }^{11}$ à questão de pesquisa adotada. Essa estratégia foi representada assim: População (cuidador informal de idosos); Intervenção (atenção à saúde do idoso); C (não se aplica); e 0 (assistência domiciliar).

Para a busca na literatura, recorreu-se a três combinações de descritores:

1. (Cuidadores) AND (Idoso OR Assistência a Idosos) AND
(Assistência Domiciliar) AND NOT (Assistência Hospitalar) AND NOT (Saúde do Idoso Institucionalizado);

2. (Cuidadores) AND (Idoso OR Assistência a Idosos) AND (Educação em Saúde) AND NOT (Saúde do Idoso Institucionalizado) AND NOT (Assistência Hospitalar);

3. (Cuidadores) AND (Idoso OR Assistência a Idosos) AND (Conhecimento) AND NOT (Assistência Hospitalar) AND NOT (Saúde do Idoso Institucionalizado).

Os artigos foram selecionados segundo critérios de inclusão que se justificam por seu intuito de levantar estudos nacionais e atuais sobre a temática em questão: a) artigos indexados nas bases de dados Lilacs e BDEnf; b) artigos disponíveis em formato eletrônico; c) artigos disponiveis em texto integral; d) artigos publicados em português; e e) artigos publicados no período de 2011 a 2016. Com a finalidade de reduzir possíveis vieses nos resultados, os critérios de exclusão foram: a) artigos de revisão bibliográfica; b) artigos de opinião; c) artigos teórico-reflexivos; e d) artigos duplicados nas bases de dados em questão.

A coleta de dados ocorreu em setembro de 2016. Para a apresentar as etapas de seleção dos artigos, utilizou-se o modelo PRISMA (Figura 1$)^{12}$.

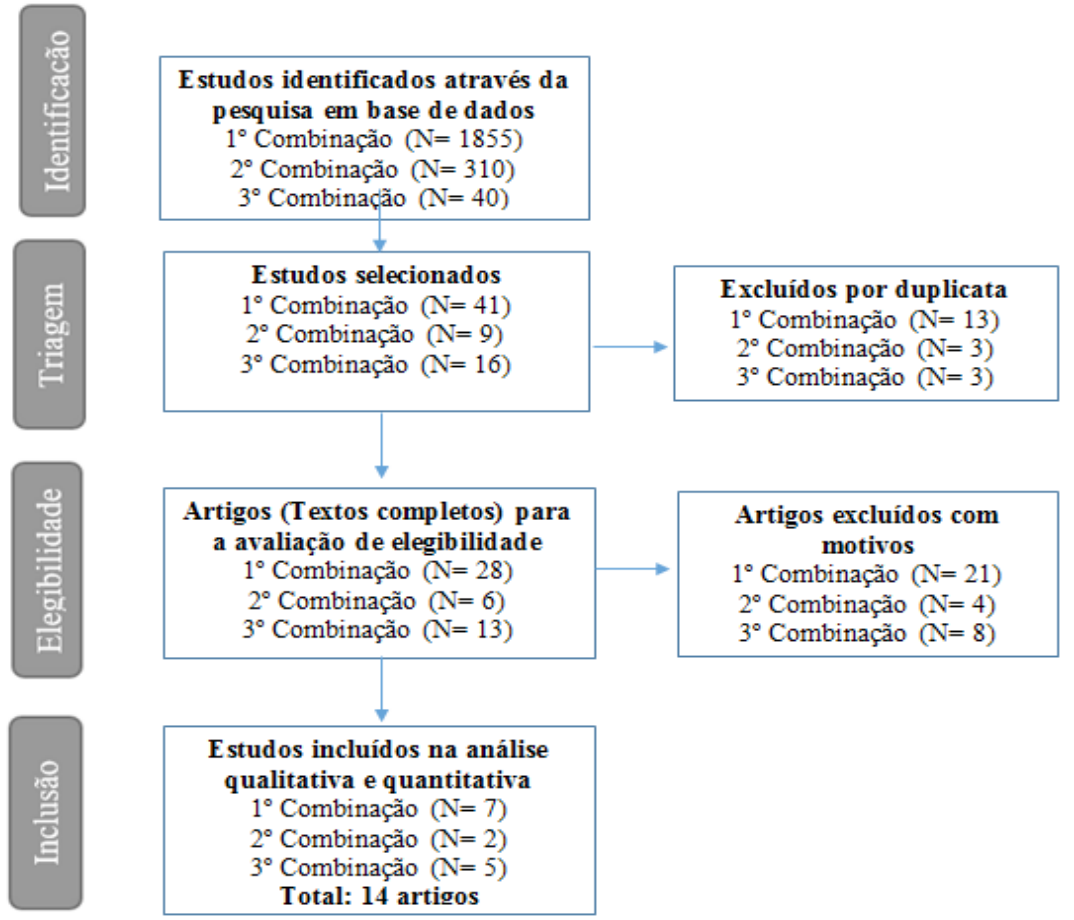

Figura 1. Fluxograma do modelo PRISMA.

Fonte: Elaborada pelas autoras.

A análise dos dados foi operacionalizada por meio de um instrumento já validado ${ }^{13}$. Recorreu-se a um quadro sinóptico elaborado para esse fim (Figura 2). A apresentação dos resultados e a discussão dos dados obtidos assumiram caráter descritivo, com base em categorias temáticas ${ }^{14}$. 


\section{RESULTADOS}

Este estudo envolveu a análise de 14 artigos. 0s anos com maior quantidade de publicações foram 2012 e 2013 (35,7\% cada), seguidos por 2011 (14,3\%) e 2014 e 2016 (7,1\% cada). Em relação à base de dados, as publicações na Lilacs assumiram destaque $(71,4 \%)$ em comparação às da $\operatorname{BDEnf}(28,6 \%)$. Quanto ao delineamento metodológico dos artigos, as abordagens que prevaleceram foram a quantitativa $(42,9 \%)$ e a qualitativa $(42,9 \%) ; 7,1 \%$ adotaram o relato de experiência e outros $7,1 \%$ utilizaram as duas abordagens (quantitativa e qualitativa).

\begin{tabular}{|c|c|c|c|c|}
\hline Autores & Título & $\begin{array}{l}\text { Ano de } \\
\text { publicação }\end{array}$ & $\begin{array}{l}\text { Base de } \\
\text { dados }\end{array}$ & Delineamento \\
\hline $\begin{array}{l}\text { Oliveira, Oliveira, Dias, } \\
\text { Rocha }^{19}\end{array}$ & $\begin{array}{l}\text { Conhecimento do cuidador sobre } \\
\text { prevenção de quedas em idosos }\end{array}$ & 2016 & BDEnf & Qualitativo \\
\hline $\begin{array}{c}\text { Lindolpho, Oliveira, } \\
\text { Sá, Brum, Valente, } \\
\text { Cruz }^{28}\end{array}$ & $\begin{array}{c}0 \text { impacto da atuação dos enfermeiros na } \\
\text { perspectiva dos cuidadores de idosos com } \\
\text { demência }\end{array}$ & 2014 & BDEnf & Qualitativo \\
\hline $\begin{array}{l}\text { Silva, Aquino, Barbosa, } \\
\text { Silva, Gomes } \\
\end{array}$ & $\begin{array}{c}\text { A perspectiva do cuidador frente ao idoso } \\
\text { com a doença de Alzheimer }\end{array}$ & 2013 & BDEnf & Qualitativo \\
\hline $\begin{array}{c}\text { Yamashita, Amendola, } \\
\text { Gaspar, Alvarenga, } \\
\text { Oliveira }{ }^{15}\end{array}$ & $\begin{array}{l}\text { Association between social support } \\
\text { and the profiles of family caregivers of } \\
\text { patients with disability and dependence }\end{array}$ & 2013 & Lilacs & Quantitativo \\
\hline $\begin{array}{l}\text { Araujo, Vidal, Brito, } \\
\text { Gonçalves, Leite, } \\
\text { Dutra, et al. }{ }^{17}\end{array}$ & $\begin{array}{c}\text { Perfil dos cuidadores e as dificuldades } \\
\text { enfrentadas no cuidado ao idoso, em } \\
\text { Ananindeua, PA }\end{array}$ & 2013 & Lilacs & Quantitativo \\
\hline $\begin{array}{l}\text { Brum, Camacho, } \\
\text { Valente, Sá, Lindolpho, } \\
\text { Louredo }^{26}\end{array}$ & $\begin{array}{l}\text { Programa para cuidadores de idosos com } \\
\text { demência: relato de experiência }\end{array}$ & 2013 & BDEnf & Relato de experiência \\
\hline $\begin{array}{l}\text { Pereira, Santos, Fhon, } \\
\text { Marques, Rodrigues }{ }^{18}\end{array}$ & $\begin{array}{c}\text { Sobrecarga dos cuidadores de idosos com } \\
\text { acidente vascular cerebral }\end{array}$ & 2013 & Lilacs & Quantitativo \\
\hline Oliveira, Caldana ${ }^{23}$ & $\begin{array}{c}\text { As repercussões do cuidado na vida do } \\
\text { cuidador familiar do idoso com demência } \\
\text { de Alzheimer }\end{array}$ & 2012 & Lilacs & Qualitativo \\
\hline Matos, Decesaro 20 & $\begin{array}{c}\text { Características de idosos acometidos pela } \\
\text { doença de Alzheimer e seus familiares } \\
\text { cuidadores principais }\end{array}$ & 2012 & Lilacs & $\begin{array}{l}\text { Quantitativo e } \\
\text { qualitativo }\end{array}$ \\
\hline Ramos, Menezes ${ }^{21}$ & $\begin{array}{l}\text { Cuidar de idosos com doença de } \\
\text { Alzheimer: um enfoque na teoria do } \\
\text { cuidado cultural }\end{array}$ & 2012 & Lilacs & Qualitativo \\
\hline $\begin{array}{l}\text { Del Duca, Martinez, } \\
\text { Bastos }\end{array}$ & $\begin{array}{c}\text { Perfil do idoso dependente de cuidado } \\
\text { domiciliar em comunidades de baixo nível } \\
\text { socioeconômico de Porto Alegre, Rio } \\
\text { Grande do Sul }\end{array}$ & 2012 & Lilacs & Quantitativo \\
\hline $\begin{array}{l}\text { Stackfleth, Diniz, } \\
\text { Fhon, Vendruscolo, } \\
\text { Fabrício-Whebe, } \\
\text { Marques, et al. }{ }^{16}\end{array}$ & $\begin{array}{l}\text { Sobrecarga de trabalho em cuidadores } \\
\text { de idosos fragilizados que vivem no } \\
\text { domicílio }\end{array}$ & 2012 & Lilacs & Quantitativo \\
\hline Cisneros, Goncalves 25 & $\begin{array}{c}\text { Educação terapêutica para diabéticos: } \\
\text { os cuidados com os pés na realidade de } \\
\text { pacientes e familiares }\end{array}$ & 2011 & Lilacs & Qualitativo \\
\hline $\begin{array}{l}\text { Rocha Junior, Corrente, } \\
\text { Hattor, Oliveira, } \\
\text { Zancheta, Gallo }\end{array}$ & $\begin{array}{l}\text { Efeito da capacitação dos cuidadores } \\
\text { informais sobre a qualidade de vida de } \\
\text { idosos com déficit de autocuidado }\end{array}$ & 2011 & Lilacs & Quantitativo \\
\hline
\end{tabular}

Figura 2. Caracterização dos artigos selecionados. Teresina, 2016.

Fonte: Elaborada pelas autoras.

Nossa revisão integrativa proporcionou a definição de categorias temáticas para a análise de nosso objeto de estudo. 


\section{Caracterização do cuidador informal no contexto domiciliar}

A produção científica analisada apresenta descrições pertinentes quanto a: sexo do cuidador; idade média; grau de parentesco; estado civil; sobrecarga de trabalho; tempo de trabalho; e escolaridade.

A associação entre as dimensões de apoio social e o perfil dos cuidadores familiares de paciente com dependência revelou que os cuidadores eram, em sua maioria, do sexo feminino $(90,0 \%)^{15}$. Outros 6 estudos corroboram o amplo predomínio do sexo feminino nas atividades envolvidas: $75 \% ; 80,7 \% ; 83,9 \% ; 90 \% ; 88,7 \%$; e $95 \%{ }^{16-21}$.

Esses dados representam o protagonismo assumido pelas cuidadoras, uma característica observada tanto em nível nacional como internacional ${ }^{15,16}$.

A respeito da idade média dos cuidadores, observou-se a média de $56,3 \operatorname{anos}^{18}$. Apesar do predomínio de adultos na função de cuidador, também se destacou a presença de grande número de idosos cuidando de outros idosos. Tal achado não é incomum nos artigos selecionados para esta revisão: a média de idade dos cuidadores variou de 40 a 62,6 anos, prevalecendo idades mais avançadas ${ }^{19}$.

Em relação ao grau de parentesco, os estudos descreveram uma distribuição entre filhos, cônjuges e genros/noras ${ }^{18}$ : a maioria dos cuidadores consistia em filhas dos idosos $(55 \%)^{21}$ e filhos $(40,7 \%)$ ou esposas $(37,0 \%)^{22}$.

No tocante ao estado civil dos cuidadores, o número de casadas predomina $(55 \%)^{21}$. 0 estado civil do cuidador se mostra relevante por poder constituir fator positivo ou negativo: trata-se de facilitador quando há apoio para as atividades envolvidas ou gera sobrecarga para o cuidador quando há acúmulo de responsabilidades ${ }^{18}$.

A sobrecarga e o tempo de trabalho estão intimamente ligados. A média de tempo como cuidador foi de 55,14 meses; dentre os cuidadores, $55 \%$ se dedicavam a portador de doença de Alzheimer em tempo integral e $45 \%$ em tempo parcial. Neste estudo, $56,3 \%$ se sentiam sobrecarregados com o cuidar e relataram cansaço, irritação e estresse e $65 \%$ dos cuidadores tinham algum problema de saúde, dentre eles hipertensão arterial, hipotireoidismo, problemas na coluna, gastrite, asma, síndrome do pânico, depressão e transtorno bipolar. Ressalta-se, ainda, que $30 \%$ deles relataram 0 surgimento da patologia após o início de suas atividades como cuidador familiar ${ }^{20}$.

$\mathrm{Na}$ avaliação da sobrecarga dos cuidadores com a escala "Burden Interview" foi observado que $8 \%$ dos cuidadores obtiveram escores $\geq 44$, um nível de sobrecarga de moderado a alto ${ }^{16}$.

Os cuidadores necessitam de revezamento ou de apoio para o cuidado do idoso, seja da própria família ou de

\section{Os cuidadores \\ necessitam de revezamento ou de apoio para o cuidado do idoso...}

profissionais da saúde, para que possam manter sua saúde e cuidar de si. Quando não se atribui o devido valor a essa condição, eles ficam sujeitos ao risco de adoecer, não pelo cuidado em si, mas pela sobrecarga à qual são submetidos ${ }^{22}$.

No que se refere à caracterização da escolaridade dos cuidados: $90 \%$ dos cuidadores sabiam ler e $85,4 \%$ sabiam escrever, sendo que $36,6 \%$ tinham Ensino Fundamental incompleto ${ }^{15}, 50 \%$ tinham Ensino Médio completo ${ }^{21}$ e $30 \%$ não haviam concluído o Ensino Fundamenta ${ }^{20}$. Há carência de instrução e isso pode trazer repercussões indesejáveis à assistência prestada ao idoso, pois são os cuidadores que colhem as orientações da equipe de saúde e sua capacidade de absorvê-las e compreendê-las depende de seu nível de entendimento ${ }^{17}$.

\section{Principais práticas do cuidador informal no contexto domiciliar}

As práticas do cuidador informal vão desde aquelas relacionadas ao bem-estar psicossocial do idoso até as atividades da vida diária - higiene corporal, higiene oral, cuidado dos cabelos, cuidado das roupas, alimentação, acompanhamento e vigilância, administração de medicações e comunicação -, ou seja, tarefas contínuas e centralizadas em uma única pessoa.

$\mathrm{Na}$ investigação das repercussões do cuidado na vida do cuidador familiar de idoso com doença de Alzheimer, observou-se que, apesar de todas as dificuldades e repercussões negativas na vida do cuidador, este tinha em mente que o compartilhamento do sofrimento com o idoso amenizava tal situação e proporcionava uma melhor condição mental desse idoso. Para os cuidadores, a promoção do bemestar psicológico do idoso era uma grande demonstração de preocupação e amor de filho - um cuidado protegido e abnegado ${ }^{23}$.

Junto com o fator psicológico, descrevem-se práticas relacionadas ao fator social, ou seja, a importância da vida social no bem-estar do idoso. 0s cuidadores relataram que um bom exemplo para a integração desse idoso à vida social é retirá-lo do ambiente domiciliar, pois até pequenos passeios em torno da casa e o encontro com vizinhos já ajuda a melhorar o humor do idoso ${ }^{24}$.

SANARE, Sobral - V.16 n.02,p.77-84, Jul./Dez. - 2017 - 81 
No tocante às atividades de vida diária, tudo começa com o amanhecer. 0s idosos acordam bem cedo, assim como seus cuidadores, para que a vigilância comece. Depois do despertar vem o banho e os cuidados relacionados à higiene; quanto maior a dependência dos idosos, maior a exigência para o cuidador (paciência, habilidade e força física). Tais atividades requerem muita observação do cuidador para detectar sinais de perigo ou de maior dependência do idoso ${ }^{21}$.

Avaliando cuidadores de pacientes com diabetes, evidenciou-se que os cuidados relativos a higiene e à detecção de lesões em um pé sensível dependem da capacidade do indivíduo examinar-se, porém, considerando que a retinopatia surge concomitantemente com a neuropatia diabética, o autocuidado dos idosos estava comprometido. A atenção do cuidador deve ser redobrada e voltada aos sinais e sintomas do idoso ${ }^{25}$. Ressalta-se, ainda, a preocupação dos cuidadores quanto às quedas - deve-se adaptar o ambiente domiciliar para preveni-las ${ }^{24}$.

Após o banho, o ato de vestir-se constitui um momento delicado - algo simples para um idoso sem doença de Alzheimer se torna um procedimento complexo para os portadores dessa doença. Abotoar um botão ou vestir uma peça íntima é uma tarefa difícil nos estágios finais da doença ${ }^{21}$.

A primeira alimentação do dia, o café da manhã, também envolve a primeira medicação. As dificuldades com a administração da medicação e alimentação são bastante relatadas pelos cuidadores. 0 idoso tem dificuldades para lembrar os horários e os tipos de medicação, não aceita os remédios e tem dificuldade para engoli-los. Quando o idoso não consegue engolir um comprimido, o cuidador recorre a outra forma de administração, diluindo, triturando ou trocando a forma de apresentação, sempre que possível21,24.

Destaca-se a recusa ou a relutância de nutrir-se, por esquecimento da última refeição, irritabilidade, dificuldade de reconhecer a fome ou algum estado de depressão. Outro ponto em destaque na literatura é a hidratação, pois os idosos se desidratam rapidamente e sua reidratação é mais lenta do que a de indivíduos mais jovens ${ }^{21,24}$.

0 momento de dormir não é só desejado, mas necessário - tanto para quem cuida como para o idoso. Geralmente, as noites do cuidador são mal dormidas, pois a vigília constante prejudica seu sono. No caso do idoso, esse momento é prejudicado pela insônia - a diminuição do sono é normal no processo de envelhecimento ${ }^{21}$.

\section{Necessidades em educação em saúde dos cuidadores informais de idosos}

Os cuidadores informais de idosos apresentam necessidades de educação em saúde quanto a: compartilhamento de saberes e práticas; processo de envelhecimento; qualidade de vida do

\section{Abotoar um botão ou vestir uma peça intima é uma tarefa difícil nos estágios finais da doença.}

idoso; compreensão do ser humano que necessita de cuidado; estímulo à autonomia do idoso; patologias específicas do idoso; desgaste físico e emocional do cuidador; e qualidade de vida do cuidador.

A educação em saúde influencia 0 modo como o cuidador reconhece e exerce seu papel. Níveis mais altos de escolaridade, participação social, recursos econômicos e condições de saúde favoráveis podem contribuir positivamente para a prática do cuidador. Entende-se que quanto maior a rede do cuidador, maior sua compreensão da importância do apoio social ${ }^{15}$.

Os cuidadores necessitam de conhecimentos, no entanto, para que as orientações proporcionadas pela educação em saúde sejam compreendidas é fundamental que os profissionais da saúde conheçam o perfil de cada cuidador e estabeleçam vínculo e bom relacionamento com cuidadores e idosos ${ }^{21,22}$.

Os profissionais da ESF são vistos como facilitadores no processo do cuidar. Revelou-se que eles se consideram atores importantes no compartilhamento de saberes e práticas. Por meio de visitas domiciliares, consultas ou atividades grupais, esses profissionais esclarecem dúvidas sobre: doenças e suas particularidades; qualidade de vida; e promoção da saúde ${ }^{21}$.

Em relação às atividades grupais, um relato de experiência do projeto denominado PRó-CUIDEM - com encontros coordenados por enfermeiras docentes em NiteróiRJ - indica que a educação em saúde proporciona: troca e construção de saberes; acolhimento das necessidades do cuidador; orientação sobre a importância das redes de apoio; e relação entre promoção da saúde e qualidade de vida ${ }^{26}$.

Tendo em vista os méritos da educação em saúde na eficácia das práticas do cuidador de idosos no contexto domiciliar, destaca-se a importância da capacitação desse cuidador ${ }^{23}$. Outro estudo destaca que os cuidadores procuram entender mais sobre a doença, não somente com as atividades de vida diária, mas buscando diversas fontes de informações em: consultas médicas; visitas domiciliares; cursos de cuidador; curso técnico em Enfermagem; meios de comunicação ${ }^{24}$.

No tocante à capacitação, avaliou-se um programa de capacitação para cuidadores informais. Em termos de 
qualidade de vida do idoso, constatou-se significativo aumento dos escores no domínio "saúde mental" e significativa diminuição dos escores no domínio “limitações por aspectos físicos". Essa diminuição pode decorrer da presença de idosos com limitações físicas e déficit de autocuidado, consequentemente, eles eram sedentários ${ }^{27}$.

A idade avançada é um fator que deve ser avaliado pelos profissionais da saúde; muitas vezes, o cuidador não apresenta condições físicas para cuidar de um idoso dependente 20 . 0 planejamento da assistência deve considerar as necessidades do cuidador para que ele não venha a adoecer e tornar-se outro ser humano que necessita de cuidados ${ }^{15}$.

No tocante às necessidades de educação em saúde por parte dos cuidadores, enfermidades específicas, como doença de Alzheimer e diabetes mellitus demandam informações sobre: sinais e sintomas da patologia; atividades voltadas às necessidades da família; estratégias para a diminuição do estresse; criação de grupos de apoio aos cuidadores; estratégias para a redução do desgaste físico e psicológico; e minimização de situações de crise, conflitos e isolamento social $20,25,28$.

Diante disso, a orientação é que os profissionais da saúde elaborem o planejamento do cuidado junto com os cuidadores $^{28}$. É de suma importância conhecer o ambiente domiciliar do cuidador, pois, para quem exerce esse papel, "viver é cuidar". Portanto, proporcionar melhor qualidade de vida ao cuidador se reflete na promoção da saúde do ser humano cuidado ${ }^{17}$.

\section{CONCLUSÃO}

Esta revisão integrativa da literatura contribui para a compreensão do objeto de estudo por meio do levantamento de práticas baseadas em evidências. Destaca-se a importância dos profissionais da saúde conhecerem o perfil e a rotina dos cuidadores familiares de pacientes com dependência, a fim de proporcionar melhor qualidade de vida para ambos no contexto domiciliar.

\section{CONTRIBUIÇÃO DAS AUTORAS}

Darleia Ferreira Barros dos Santos contribuiu com 0 delineamento e a realização da pesquisa. Elane Barreira de Carvalho contribuiu com a análise e interpretação dos dados. Maria do Perpétuo Socorro Santiago do Nascimento contribuiu com a interpretação dos dados e a redação do manuscrito. Daniele Martins de Sousa contribuiu com a redação e revisão crítica do manuscrito. Herica Emilia Félix de Carvalho contribuiu com a interpretação dos dados e a revisão crítica do manuscrito.

\section{REFERÊNCIAS}

1. Brasil. Tábuas completas de mortalidade do Brasil de 2013 [document on the internet]. Rio de Janeiro: IBGE; 2013 [cited 2017 Dec 7]. Available from: http://www.ibge.gov.br/ home/estatistica/populacao/tabuadevida/2013/default.shtm

2. Carvalho MP, Luckow ELT, Siqueira FV. Quedas e fatores associados em idosos institucionalizados no município de Pelotas (RS, Brasil). Ciênc Saúde Colet [serial on the internet]. 2011 [cited 2016 Sep 29];16(6):2945-52. Available from: http://www.scielo.br/scielo.php?script=sci arttext\&pid=S1413-81232011000600032

3. Lima-Costa MF, Barreto S, Giatti L, Uchôa E. Desigualdade social e saúde entre idosos brasileiros: um estudo baseado na Pesquisa Nacional por Amostra de Domicilios. Cad Saúde Pública [serial on the internet]. 2003 [cited 2016 Sep 29];19(3):745-57. Available from: http:// www.scielo.br/scielo.php?script $=$ sci arttext\&pid=S0102311X2003000300007\&lng=en

4. Anjos KFD, Boery RNSD0, Pereira R., Santos VC, Boery EN, Casotti CA. Perfil de cuidadores familiares de idosos no domicílio. Rev Pesqui Cuid Fundam (Online) [serial on the internet]. 2014 [cited 2016 Sep 29];6(2):45061. Available from: http://www.seer.unirio.br/index.php/ cuidadofundamental/article/view/3083/pdf 1224

5. Eliopoulos C. Enfermagem gerontológica. 5. ed. Porto Alegre: Artmed; 2005.

6. Vieira CPB, Fialho AVM, Freitas CHA, Jorge MSB. Práticas do cuidador informal do idoso no domicílio. Rev Bras Enferm [serial on the internet]. 2011 [cited 2016 Sep 29];64(3):570-9. Available from: http://www.scielo.br/scielo.php?script=sci arttext\&pid=S0034-71672011000300023\&lng=en

7. Santiago RF, Luz MHBA. Práticas de educação em saúde para cuidadores de idosos: um olhar da enfermagem na perspectiva freireana. REME Rev Min Enferm [serial on the internet]. 2012 [cited 2016 Sep 29];16(1):136-42. Available from: file:///D:/v16n1a19.pdf

8. Brasil. Manual do cuidador da pessoa idosa [document on the internet]. Brasília (DF): Ministério da Saúde; 2008 [cited 2017 Dec 7]. Available from: http://www.sdh.gov. br/assuntos/pessoa-idosa/legislacao/pdf/manual-docuidadora-da-pessoa-idosa

9. Muniz EA, Freitas CASL, Albuquerque IMAN, Linhares MSC. Assistência domiciliar ao idoso no contexto da Estratégia Saúde da Família: análise da produção científica. Sanare (Sobral, 0nline) [serial on the internet]. 2014 [cited 2016 Sep 29];13(2):86-91. Available from: https://sanare. emnuvens.com.br/sanare/article/view/578/311

10. Botelho LLR, Cunha CCA, Macedo M. 0 método da revisão integrativa nos estudos organizacionais. Gestão e Sociedade [serial on the internet]. 2011 [cited 2016 Sep 29];5(11):12136. Available from: http://www.gestaoesociedade.org/ 
11. Santos CMC, Pimenta CAM, Nobre MRC. A estratégia PICO para a construção da pergunta de pesquisa e busca de evidências. Rev Latinoam Enferm [serial on the internet]. 2007 [cited 2016 Sep 29];15(3):508-11. Available from: http:// www.scielo.br/scielo.php?script $=$ sci arttext\&pid=S0104$\underline{11692007000300023 \& \operatorname{lng}=\text { en }}$

12. Fuchs SC, Paim BS. Revisão sistemática de estudos observacionais com metanálise. Rev HCPA \& Fac Med Univ Fed Rio Gd do Sul [serial on the internet]. 2010 [cited 2016 Oct 30];30(3):294-301. Available from: http://seer.ufrgs.br/ index.php/hcpa/article/view/16551/9849

13. Ursi ES, Gavão CM. Prevenção de lesões de pele no perioperatório: revisão integrativa da literatura. Rev Latinoam Enferm [serial on the internet]. 2006 [cited 2016 Sep 29];14(1):124-31. Available from: http:// www.scielo.br/scielo.php? script $=$ sci arttext\&pid=S0104$11692006000100017 \& \operatorname{lng}=$ en

14. Minayo MCS. 0 desafio do conhecimento: pesquisa qualitativa em saúde. 12. ed. São Paulo: Hucitec; 2010.

15. Yamashita CH, Amendola F, Gaspar JC, Alvarenga MRM, Oliveira MAC. Association between social support and the profiles of family caregivers of patients with disability and dependence. Rev Esc Enferm USP [serial on the internet]. 2013 [cited 2016 Sep 29];47(6):1359-66. Available from: http:// www.scielo.br/scielo.php?script $=$ sci arttext\&pid=S0080$\underline{62342013000601359 \& \operatorname{lng}=e n}$

16. Stackfleth R, Diniz MA, Fhon JRS, Vendruscolo TRP, Fabrício-Whebe SCC, Marques $S$, et al. Sobrecarga de trabalho em cuidadores de idosos fragilizados que vivem no domicílio. Acta Paul Enferm [serial on the internet]. 2012 [cited 2016 Sep 29];25(5):768-74. Available from: http:// www.scielo.br/scielo.php?script $=$ sci arttext\&pid=S0103$\underline{21002012000500019 \& \operatorname{lng}=e n}$

17. Araujo JS, Vidal GM, Brito FN, Gonçalves DCA, Leite DKM, Dutra CDT, et al. Perfil dos cuidadores e as dificuldades enfrentadas no cuidado ao idoso, em Ananindeua, PA. Rev Bras Geriatr Gerontol [serial on the internet]. 2013 [cited 2016 Sep 29];16(1):149-58. Available from: http:// www.scielo.br/scielo.php? script $=$ sci arttext\&pid=S1809$\underline{98232013000100015 \& \operatorname{lng}=e n}$

18. Pereira RA, Santos EB, Fhon JRS, Marques S, Rodrigues RAP. Sobrecarga dos cuidadores de idosos com acidente vascular cerebral. Rev Esc Enferm USP [serial on the internet]. 2013 [cited 2016 Sep 29];47(1):185-92. Available from: http:// www.scielo.br/scielo.php?script $=$ sci arttext\&pid=S0080$\underline{62342013000100023 \& \operatorname{lng}=e n}$

19. Oliveira PP, Oliveira ACD, Dias AR, Rocha FCV. Conhecimento do cuidador sobre prevenção de quedas em idosos. Rev Enferm UFPE On Line [serial on the internet]. 2016 [cited 2016 Sep 29];10(2):585-92. Available from: http://www.revista.ufpe.br/revistaenfermagem/index.php/ revista/article/view/7073/pdf 9604

20. Matos PCB, Decesaro MN. Características de idosos acometidos pela doença de Alzheimer e seus familiares cuidadores principais. Rev Eletrônica Enferm [serial on the internet]. 2012 [cited 2016 Sep 29];14(4):857-65. Available from: http://www.fen.ufg.br/revista/v14/n4/v14n4a14.htm

21. Ramos JLC, Menezes MDRD. Cuidar de idosos com doença de Alzheimer: um enfoque na teoria do cuidado cultural. Rev Rene [cited 2016 Sep 29];13(4):805-15. Available from: http://www.periodicos.ufc.br/index.php/rene/article/ view/4037

22. Del Duca GF, Martinez AD, Bastos GAN. Perfil do idoso dependente de cuidado domiciliar em comunidades de baixo nível socioeconômico de Porto Alegre, Rio Grande do Sul. Ciênc Saúde Colet [serial on the internet]. 2012 [cited 2016 Sep 29];17(5):1159-65. Available from: http:// www.scielo.br/scielo.php?script $=$ sci $\operatorname{arttext\& pid=S1413-}$ $81232012000500010 \& \operatorname{lng}=e n$

23. Oliveira APP, Caldana RHL. As repercussões do cuidado na vida do cuidador familiar do idoso com demência de Alzheimer. Saúde Soc [serial on the internet]. 2012 [cited 2016 Sep 29];21(3):675-85. Available from: http:// www.scielo.br/scielo.php?script $=$ sci_arttext\&pid=S0104$12902012000300013 \& \operatorname{lng}=$ en

24. Silva SPN, Aquino CAG, Barbosa TLA, Silva CSO, Gomes LMX. A perspectiva do cuidador frente ao idoso com a doença de Alzheimer. Rev Pesqui Cuid Fundam (Online) [serial on the internet]. 2013 [cited 2016 Sep 29];5(1):3333-42. Available from: http://www.redalyc.org/pdf/5057/505750897018.pdf

25. Cisneros LL, Gonçalves LAO. Educação terapêutica para diabéticos: os cuidados com os pés na realidade de pacientes e familiares. Ciênc Saúde Colet [serial on the internet]. 2011 [cited 2016 Sep 29];16(Suppl 1):1505-14. Available from: http://www.scielo.br/scielo.php?script=sci arttext\&pid=S1413-81232011000700086\&lng=en

26. Brum AKR, Camacho ACLF, Valente GSC, Sá SPC, Lindolpho MC, Louredo DS. Programa para cuidadores de idosos com demência: relato de experiência. Rev Bras Enferm [serial on the internet]. 2013 [cited 2016 Sep 29];66(4):619-24. Available from: http://www.scielo.br/scielo.php?script=sci arttext\&pid=S0034-71672013000400025\&lng=en

27. Rocha Júnior PR, Corrente JE, Hattor CH, Oliveira IM, Zancheta D, Gallo CG, et al. Efeito da capacitação dos cuidadores informais sobre a qualidade de vida de idosos com déficit de autocuidado. Ciênc Saúde Colet [serial on the internet]. 2011 [cited 2016 Sep 29];16(7):3131-7. Available from: http://www.scielo.br/scielo.php?script=sci arttext\&pid $=$ S1413-812320110000800013\&lng $=$ en

28. Lindolpho MC, Oliveira JBde, Sá SPC, Brum AK, Valente GSC, Cruz TJPda. 0 impacto da atuação dos enfermairos na perspectiva dos cuidadores de idosos com Demência. J. res.: fundam. care. Online [serial on the internet]. 2014 [cited 2016 Sep 29]; 6(3):1078-1089. Available from: http:// www.seer.unirio.br/index.php/cuidadofundamental/article/ view/3452/pdf 1360

Recebido em 09/02/2017 Aprovado em 20/07/2017 